\title{
Reliability analysis of integrated transmission based on fuzzy multi-state fault tree
}

\author{
Zhang Jinle ${ }^{1, a}$, Peng Wensheng ${ }^{2, b}$, Zhang Jianguo ${ }^{2}$ and Guo Jing ${ }^{1}$ \\ ${ }^{1}$ Science and technology on vehicle transmission lab, China north vehicle institute, Beijing, China \\ ${ }^{2}$ School of Reliability and Systems Engineering, Beihang University, Beijing, China \\ a120108980@qq.com, bwshpeng@buaa.edu.cn
}

Keywords: Integrated transmission, multi-state reliability, fault tree, fuzzy theory, cut-set level

\begin{abstract}
Integrated transmission is the key part of heavy vehicles, and its reliability could impact the performance of the vehicles directly. This paper proposes a fuzzy multi-state fault tree method for reliability analysis of the integrated transmission system. On the one hand, the multi-state logic tree can describe the complex failure states of the integrated transmission. On the other hand, most important empirical data and subjective information can be used with the help of the fuzzy theory, and it could avoid the waste of information in reliability analysis. The degradation performance of some parts in the integrated transmission is also considered in the reliability analysis. The multi-state fault tree is used to build the reliability model of the integrated transmission, and the fuzzy cut-set technique is used to calculate the reliability parameters of the fault tree. The application demonstrates that the method proposed in this paper could get good results in the integrated transmission reliability analysis.
\end{abstract}

\section{Introduction}

Integrated transmission of heavy vehicle can integrate the functions of transmission, direction changing and speed changing, and these functions match each other. With all components assembled in a box, the integrated transmission has the features of compact structure, small size and high integration. Therefore the integrated transmission technology is widely used in high-speed tracked vehicles. The parts of the integrated transmission can be divided into three categories: mechanical parts, hydraulic control parts and electronic control parts. The reliability of integrated transmission has following features: (1) there are many reliability units covering electronic units, mechanical units and hydraulic units, these units have different reliability levels and different reliability distributions; (2) most of the reliability units cannot be described as two states of "success" and "failure", instead, these reliability units have degradation characters and multi failure states. Nowadays few failure data of the integrated transmission have been collected, the reliability analysis is even less. Z[1] analyzes integrated transmission based on the FTA_FMEA; X[2] proposes entropy weight fuzzy comprehensive evaluation on failure mode criticality of integrated transmission system; X[3] design the expert system for the integrated transmission; $\mathrm{W}[4]$ and $Z[5]$ have done some work of the reliability analysis for the gear and gasket ring of the integrated transmission.

Fault tree analysis (FTA) was first proposed by the Watson in 1961, and this method has been widely used in the aviation, aerospace, nuclear, electric and other engineering fields [6-11]. Traditional fault tree is a logic block method, and its objective is finding all probable failure reasons of the top event. The analysis foundation is the "two-state" assumption of 'failure' and 'success'. However, many events are not "two-state" in the real engineering, so the traditional two-state fault tree model is not suitable in most real engineering cases, especially for the integrated transmission analysis. The reliability analysis of the integrated transmission has follow features: on the one hand, there is much subjective information in the integrated transmission, such as expert knowledge, historical information. On the other hand, there are degradation characters in it, especially for the mechanical parts. This paper proposes a fuzzy multi-state FTA method considering the degradation 
performance and epistemic uncertainty information of the integrated transmission, and the minimal path set method is utilized for reliability calculation.

\section{Multi-state fault tree}

The multi-state fault tree proposed in this paper is the improved model of traditional fault tree. The multi-state event and multi-state logic gate are defined before modeling multi-state fault tree.

- Definition 1 (multi-state event)

If there are some other states between "safe" and "failure" in one event, then this event is defined as multi-state event.

- Definition 2 (multi-state logic gate)

If the input events of a logic gate contain at least one multi-state event, then this logic gate is defined as multi-state logic gate.

Respectively, the fault tree is defined as a multi-state fault tree if there is at least one multi-state event in it.

The guideline of modeling the multi-state fault tree is that all possible failure modes is classified from the top event level by level, then the failure components corresponding to the failure modes is identified in the system. The failure modes and failure components are classified as different state events, and the multi-state logic gates are used to link these events. It must be noted that the system should be divided into sub-systems without joint sets, and failure mode of different sub-systems should be independent on each other. A typical multi-state fault tree model is as shown in Fig. 1.

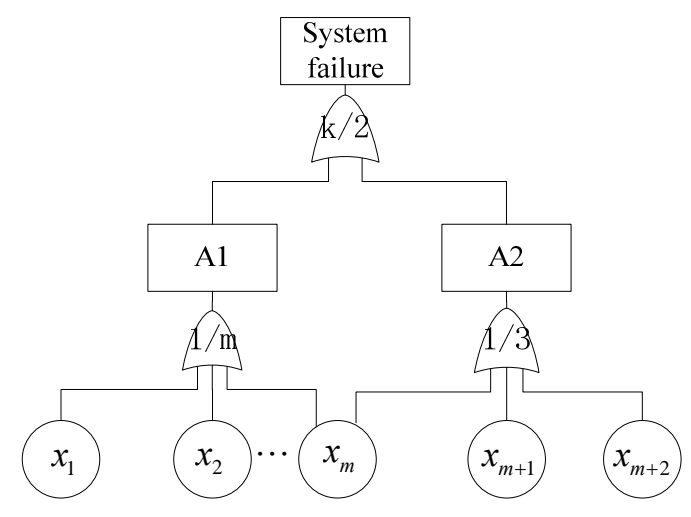

Fig 1. A multi-state fault tree

The state definition of the output events is based on the multi-state vote gate $k / n$. Assuming that there are $n$ input events $X_{i},(i=1,2, \ldots, n)$. The state of $X_{i}$ satisfies $S_{X_{i}} \in\{0,0.5,1\}$, then the state of the output event $Y$ can be defined as follow:

$$
S_{Y}= \begin{cases}0 & \sum_{i=1}^{n} S_{X_{i}}=0 \\ 0.5 & 0<\sum_{i=1}^{n} S_{X_{i}}<0.5 k, S_{X_{i}} \leq 0.5 \\ 1 & \text { otherwise }\end{cases}
$$

\section{Fuzzy theory and fuzzy multi-state fault tree model}

In fuzzy set theory, the elements are not determined to belong to a set or not. The degree of membership is described by $\mu_{A}(x)$ between in $[0,1]$, and it presents the degree of elements $x$ belong to a set $A$. The fuzzy set is uncertain, and it has no certain range, so the mathematic is useful if only the fuzzy sets were transferred into the crisp sets. The cut-set level technique plays an important role in the transfer procession. 
Defining a fuzzy set $A$ in the a discourse domain, for any $\alpha \in[0,1]$, the set $A_{\alpha}=\left\{u \mid \mu_{A} \geq \alpha, u \in U\right\}$ is the cut level $\alpha$ of $A$. For example, for a triangle membership function $\mu_{\overparen{A}}$,as in Eq. 2:

$$
\mu_{A_{A}}(x)=\left\{\begin{array}{cl}
\frac{x-a}{b-a} & a<x<b \\
1 & x=b \\
\frac{c-x}{c-b} & b<x<c
\end{array}\right.
$$

Then the $\alpha$ cut set of the fuzzy set is $\left[a^{(\alpha)}, c^{(\alpha)}\right]$, as in Fig. 2. Different cut-set levels have different cut-set intervals.

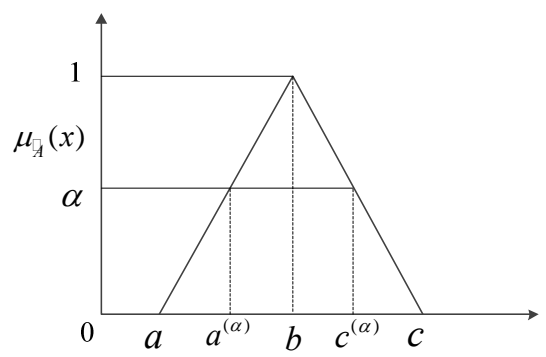

Fig 2. The cut-level of the triangle membership function

Based on the definition of the multi-state fault tree in the section 2, the fuzzy multi-state fault tree could be modeled. The key point of the fuzzy multi-state fault tree is combining the fuzzy set theory and the multi-state fault tree. Thus the subjective uncertainty and the historical data could be used in the reliability analysis in the engineering application. The detail operations as follows: the fuzzy membership function is attached in the basic events of the multi-state fault tree, and the fuzzy membership could be defined according to the historical data; for reliability analyzing, the cut-set approach is employed to transfer the fuzzy set to crisp set, then the set theory could be used to calculate the reliability of the multi-state fault tree.

\section{Reliability analysis of the integrated transmission}

A. System analysis of the integrated transmission

The basic functions of the integrated transmission are: transmission, change direction, change speed. The function modules are as shown in Fig. 3.

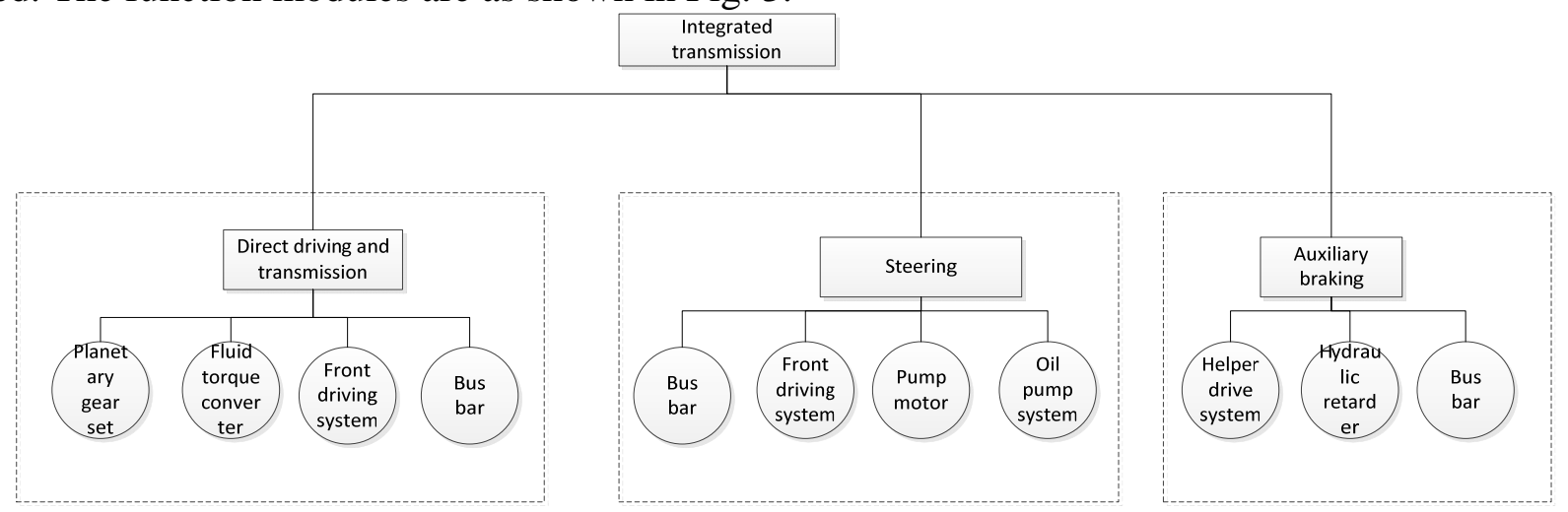

Fig 3. Functional decomposition of the integrated transmission

The information of sub-systems is shown in Table 1, including their numbers, names and components. 
Table 1. The structure of the integrated transmission

\begin{tabular}{|l|l|l|}
\hline No. & \multicolumn{1}{|c|}{ Sub-systems } & \multicolumn{1}{c|}{ Components } \\
\hline E1 & Planetary transmission & Planetary gear, axle shaft, bearing, brake \\
\hline E2 & Fluid torque converter & Pump wheel, stato, lockup clutch ,turbo \\
\hline E3 & Front driving mechanism & Bevel gear, straight gear, bearing \\
\hline E4 & Bus bar & Planetary gear, sun gear, steering gear, oil seal \\
\hline E5 & Pump motor & Casing, gear, valve plate, trunk piston, swash plate \\
\hline E6 & Oil pump system & Oil pump, transfer pipe \\
\hline E7 & Helper drive system & Driving gear, driven gear, bearing \\
\hline E8 & Viscous clutch & $\begin{array}{l}\text { Main shaft, shield ring, piston, spring, friction disk, spline } \\
\text { hub }\end{array}$ \\
\hline
\end{tabular}

Integrated transmission is a complex mechatronic-hydraulic system, it has many failure modes. Since the failure modes of most parts in integrated transmission have the characters of degradation, these failure modes (such as the wear of the gear, the leakage of the oil pump and so on) could be described as "multi-state" events as mentioned in section 2. So the multi-state fault tree model of the integrated transmission is as shown in Fig. 4. Table 2 lists the failure modes of the integrated transmission.

Table 2. Failure modes of integrated transmission

\begin{tabular}{|l|l|}
\hline \multicolumn{1}{|c|}{ failure modes } & \multicolumn{1}{|c|}{ code } \\
\hline small power of steering pump & F101 \\
\hline no output of steering linkages & F102 \\
\hline no output of bus bar & F103 \\
\hline small power of the front driving mechanism & F104 \\
\hline the output of the Planetary transmission is not stable & F105 \\
\hline leak of the oil box & F201 \\
\hline oil plug & F202 \\
\hline wear of the hydrovalve & F203 \\
\hline spring deformation & F204 \\
\hline piston clearance become large & F205 \\
\hline crack of the main shaft & F206 \\
\hline wear of the steering gear & F207 \\
\hline wear of the planetary gear & F208 \\
\hline casing deformation & F209 \\
\hline crack of the friction plate & F210 \\
\hline turbo deformation & F211 \\
\hline wear of the bear & F212 \\
\hline small power of the Pump motor & F213 \\
\hline crack of the brake & F214 \\
\hline crack of the shield ring & F215 \\
\hline
\end{tabular}

Through the expert knowledge and historical data of the integrated transmission, the state classification of the subsystems of the integrated transmission and their probabilistic estimator are determined as in Table 3. 


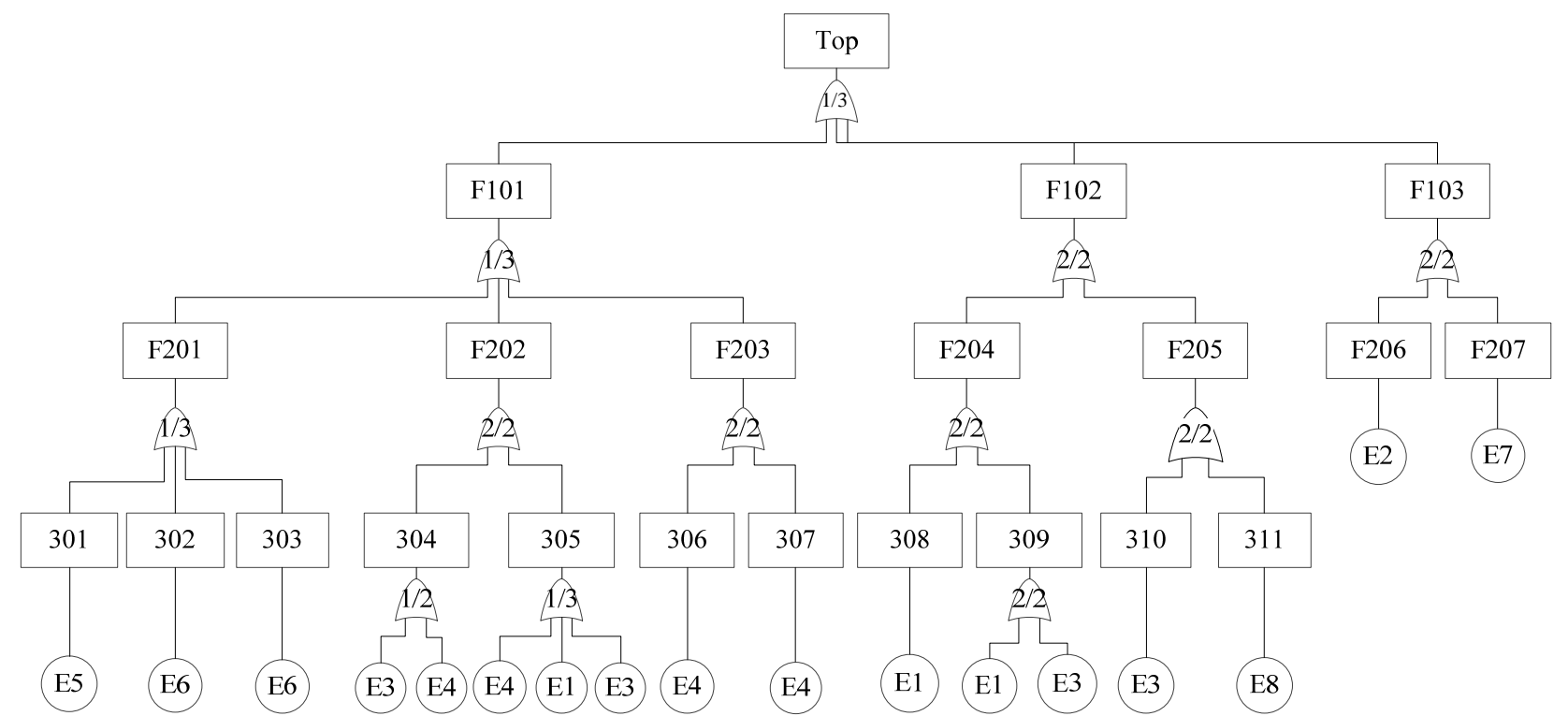

Fig 4. Multi-state fault tree of integrated transmission

Table 3. State probabilities of sub-systems

\begin{tabular}{|c|c|c|c|c|c|c|c|}
\hline \multirow{2}{*}{$\begin{array}{c}\text { Sub- } \\
\text { systems }\end{array}$} & \multicolumn{3}{|c|}{ state } & \multirow{2}{*}{$\begin{array}{c}\text { Sub- } \\
\text { systems }\end{array}$} & \multicolumn{3}{|c|}{ state } \\
\hline & & 0.5 & 1 & & 0 & 0.5 & 1 \\
\hline E1 & 0.98 & 0.010 & 0.010 & E5 & 0.95 & 0.050 & 0.010 \\
\hline $\mathrm{E} 2$ & 0.9 & 0.010 & 0.01 & E6 & 0.96 & 0.0 & 0.02 \\
\hline E3 & 0.98 & 0.010 & 0.02 & E7 & 0.98 & 0.00 & 0.001 \\
\hline $\mathrm{E} 4$ & 0.99 & 0.030 & 0.01 & E8 & 0.98 & 0.010 & 0.010 \\
\hline
\end{tabular}

The definition of the fuzzy membership functions of the bottom events. According to the engineering experience, the fuzzy membership function $\mu_{\left(x_{i}\right)}$ of the bottom events could be defined as the isosceles triangles function, and the span of left/right side is $15 \%$.

The fuzzy membership function of the reliability is defined as:

$$
\mu_{(x)}= \begin{cases}\frac{x-0.85}{0.15} & 0.85<x<1 \\ 1 & x=1 \\ \frac{1.15-x}{0.15} & 1<x<1.15\end{cases}
$$

B. Calculation of the reliability

The reliability calculation of the FTA is the process that calculating the state probability of the middle event and top events based on the state probability of the bottom events(basic events). In multi-state fault tree of the integrated transmission, the reliability of the multi-state event $E$ could be defined as :

$$
R_{E}=P\left(S_{E}=0\right)+P\left(S_{E}=0.5\right)=1-P\left(S_{E}=1\right)
$$

Minimal path set based method is an important technique for system reliability analysis. The detail steps of this method are as follows:

Assuming multi-state system is composed of $m$ independent subsystems $X_{i}(i=1,2, \cdots, m)$, the system sate is $S_{T}$ and its $k$ minimal paths are $z_{1}, z_{2}, \cdots, z_{k}$, then the minimal path matrix is:

$$
\mathbf{C}=\left(\begin{array}{cccc}
1 & 1 & \cdots & 1 \\
z_{1} & z_{2} & \cdots & z_{k}
\end{array}\right)
$$


Q is the $(m+1) \times\left(\begin{array}{l}k \\ j\end{array}\right)$ matrix obtained from the matrix $C, X_{i}$ has $M_{i}+1$ discrete states and all of them are mutex. The discrete states satisfy $S_{X_{i}} \in\left\{0,1, \cdots, M_{i}\right\}$, the states probabilities are given , then the reliability of the system state $S_{T}$ is

$R_{T}=-\sum_{j=1}^{k} f\left(\mathbf{C}_{j}\right) \otimes g\left(\mathbf{C}_{j}\right)$

The detail calculation rules could be seen in reference [12].

For reliability calculation of the integrated transmission, we choose 6 different cut-set levels of 0,0.2,0.4,0.6,0.8,1 in fuzzy FTA. Fig. 5 shows the five different cut levels of the membership function, and then different crisp intervals could be obtained to calculate the reliability.

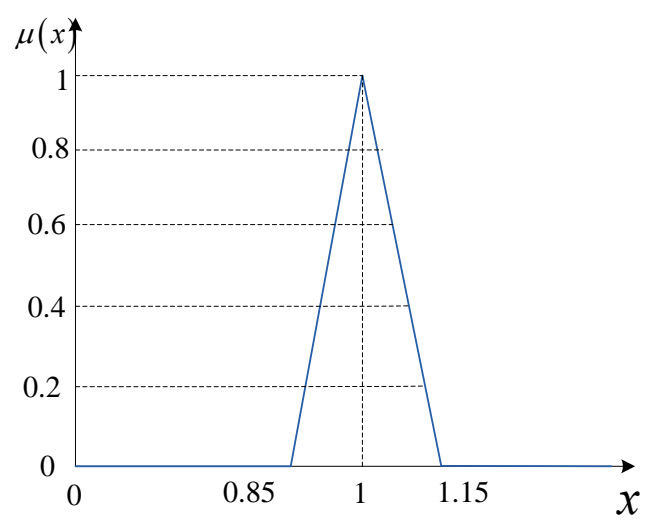

Fig 5. Fuzzy membership function of integrated transmission

According to the Fig. 4, the system reliability of the integrated transmission is

$R_{\text {Top }}=R_{101} R_{102} R_{103}$

For event $F_{101}$, there are 5 minimal cut sets . So the $\mathbf{C}_{\mathrm{F}_{101}}$ is :

$\mathbf{C}_{\mathrm{F}_{101}}=\left[\begin{array}{ccccc}1 & 1 & 1 & 1 & 1 \\ 0 & 0 & 0 & 0 & 0 \\ 0 & 0 & 0.5 & 0 & 0 \\ 0 & 0 & 0 & 0.5 & 0 \\ 0 & 0 & 0 & 0 & 0.5\end{array}\right]^{\mathrm{T}}$

And then $\mathbf{C}_{\mathrm{F}_{102}}$ and $\mathbf{C}_{F_{103}}$ are:

$$
\begin{aligned}
\mathbf{C}_{\mathrm{F}_{102}} & =\left[\begin{array}{ccc}
1 & 1 & 1 \\
0 & 0.5 & 0 \\
0 & 0 & 0.5
\end{array}\right]^{\mathrm{T}} \\
\mathbf{C}_{\mathrm{F}_{103}} & =\left[\begin{array}{cc}
1 & 1 \\
0 & 0.5
\end{array}\right]^{\mathrm{T}}
\end{aligned}
$$

The calculation of the fuzzy membership function. Based on the fuzzy membership functions of all bottom events in the fault tree, the membership function of the top event could be obtained by solving the following optimization models, the particle swarm optimization (PSO) algorithm is employed to solving this model in this paper because the optimization model is high nonlinearity. The detail of PSO could be seen in reference [13].

Minimize/Maximize: $\quad R\left(X_{i}\right)$

subject to :

$$
\begin{aligned}
& \mu\left(X_{i}\right) \geq \alpha, \\
& 0 \leq \alpha \leq 1,
\end{aligned}
$$


After optimization, the fuzzy membership function of the system could be seen in Fig. 6 . The reliability at different cut-set levels are as in Table 4. The Fig. 6 shows that the optimization based method could achieve a better result compared to the interval arithmetic. The span width of the membership function of the system reliability obtained by the PSO algorithm is much smaller than the interval arithmetic (IA), when the $\mu(x)=0$, the cut set interval range is largest, the PSO result is 0.1304 , the IA result is 0.0611 . When the $\mu(x)=1$, the cut set interval is 0 , so the result obtained by the PSO and IA is the same. It also means that the fuzzy set is also crisp set when $\mu(x)=1$. From the Table 4, different cut levels gives the system reliability respectively, it is meaningful for the decision-making .

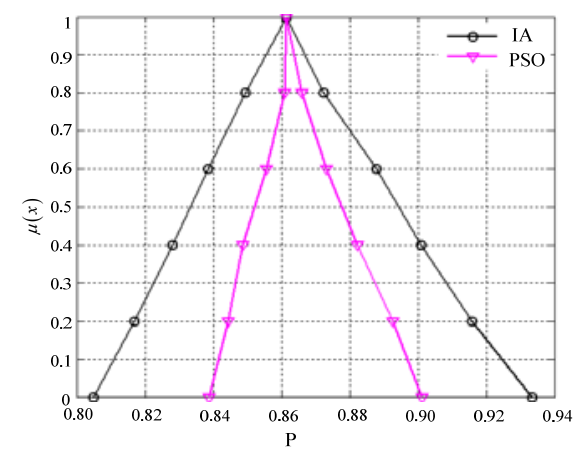

Fig 6. Final fuzzy membership function of system

Table 4. System reliability at different cut set levels

\begin{tabular}{|l|l|}
\hline \multicolumn{1}{|c|}{ Cut set level $\alpha$} & \multicolumn{1}{c|}{ Reliability } \\
\hline 0 & $0.840342,0.902342$ \\
\hline 0.2 & 0.842134 .0 .888631 \\
\hline 0.4 & $0.850852,0.881324$ \\
\hline 0.6 & $0.852513,0.871912$ \\
\hline 0.8 & $0.861217,0.868787$ \\
\hline 1 & 0.86221 \\
\hline
\end{tabular}

\section{Summary}

This paper proposes a fuzzy multi-state fault tree analysis method and applies it in the reliability analysis of integrated transmission. The cognition uncertainty and other subjective information could be used in the design of the devices based on the fuzzy theory. The fuzzy multi-state fault tree method could overcome the shortcoming of the lacking of probabilistic information, and it also concerns on the degradation character of the mechanical parts in the integrated transmission. The main contributions of this paper are:

- The fuzzy theory could make use of both subjective and objective information of the integrated transmission

- The multi-state FTA concerns on the degradation character of the mechanical parts in the integrated transmission

- The fuzzy multi-state FTA method is more meaningful in the engineering application, and make reliability more reasonable. 


\section{References}

[1] Z. Yongfeng, L. Feng, L. Liang, Zh. Lei. "Fault analysis on integrated transmission equipment of tracked vehicle based on FTA-FMEA,” J. Agricultural Equipment \& Vehicle Engineering. vol. 51, (2013), p. 61-63.

[2] X. Baorong, L. Rongli, W. Huoming et al. "Entropy weight fuzzy comprehensive evaluation on failure mode criticalityof integrated transmission system,"J.Acta Armamentarii, vol. 34(11) (2013),p.1380-1386.

[3] X. Feng,Y. Qingdong,W. Wei, “The design of fault diagnose expert system for armored vehicle power train,”J. Modern manufacturing engineering ,vol.3, (2008), p.105-108.

[4] W Wenqing, Z. Mu-qiao. "Analysis and calculation of fuzzy reliability on the cast ironsealing ring in composite transmissions,”J. Transactions of Beijing Institute of Technology. Vol.25, (2005), p.112-115.

[5] L. Chenxi, C. Nan, Y. Jianing. "Reliability analysis of servo turret based on multi-state fault tree,”J. Journal of Southest University (Natural Science Edition), vol.44, (2014), p.538-543.

[6] Z. Xingxiang, F. Xinhai, Y. Chiwei. "Research on gear fault diagnosis of integrated transmission,” J. Mechanical Transmission, (2013), p.90-93.

[7] H A Watson, "Launch control safety study" . Murray Hill, USA: Bell Telephone Laboratories,(1961),p.131-189.

[8] A. Volkanovski, M. Cepin, B Mavko. “Application of the fault tree analysis for assessment of power system reliability,”J. Reliability Engineering and System Safety, vol.94, (2009) , p.1116-1127.

[9] A Mentes, I H Helvacioglu. "An application of fuzzy fault tree analysis for spread mooring systems,” J. Ocean Engineering, vol.38, (2011), p. 285-294.

[10] F A Rahman, A Varuttamaseni, M Kintner Meyer, et al. "Application of fault tree analysis for customer reliability assessment of a distribution power system,” J. Reliability Engineering and System Safety, vol. 111,( 2013), p.76-85.

[11] Z. Liang, G. Xin, “Method of fault tree construction for multistate system,” J. Journal of System Engineering. vol.23,(1998),p.74-78.

[12] Z Guozhi. "Reliability research of complex systems". Beijing, College of Applied Science. Beijing University of Technology. (2009).

[13] Gargn, S.P.Sharma, "Stochastic behavior analysis of complex repairable industrial systems utilizing uncertain data,”J. ISA Transactions,vol. 51,(2012), p.752-762. 